\title{
CONTINUIDADE DO CUIDADO E O INTERACIONISMO SIMBÓLICO: UM ENTENDIMENTO POSSÍVEL
}

\author{
Fernanda Catafesta Utzumi ${ }^{1}$, Maria Ribeiro Lacerda ${ }^{2}$, Elizabeth Bernardino ${ }^{3}$, Ingrid Meireles Gomes ${ }^{4}$, Gisele \\ Knop Aued ${ }^{5}$, Solange Meira de Sousa
}

${ }^{1}$ Doutoranda do Programa de Pós-Graduação em Enfermagem (PPGENF), da Universidade Federal do Paraná (UFPR).
Enfermeira da Unidade de Saúde Ocupacional e Acessibilidade UFPR Campus Jandaia do Sul. Curitiba, Paraná, Brasil. E-mail:
fernandacatafesta@yahoo.com.br
${ }^{2}$ Doutora em Enfermagem. Professora do PPGENF/UFPR. Curitiba, Paraná, Brasil. E-mail: mrlacerda55@gmail.com
${ }^{3}$ Doutora em Enfermagem. Professora do PPGENF/UFPR. Curitiba, Paraná, Brasil. E-mail: elizaber@ufpr.br
${ }^{4}$ Doutoranda do PPGENF/UFPR. Enfermeira do Hospital das Clínicas da UFPR. Curitiba, Paraná, Brasil. E-mail: inguide@
gmail.com
${ }_{5}^{5}$ Doutoranda do PPGENF/UFPR. Curitiba, Paraná, Brasil. E-mail: giseleknop@gmail.com
${ }^{6}$ Doutoranda do PPGENF/UFPR. Curitiba, Paraná, Brasil. E-mail: solangesmj@gmail.com

RESUMO

Objetivo: buscar um entendimento pela consubstanciação entre o conceito de continuidade do cuidado ao referencial teórico do interacionismo simbólico, no sentido de agregar uma possibilidade de fortalecê-lo como conceito indispensável ao cuidado prestado aos usuários do sistema de saúde e provocar uma necessária reflexão sobre este.

Método: estudo teórico-reflexivo, organizado em quatro seções, sobre a continuidade do Cuidado analisado sob a luz do referencial do interacionismo simbólico.

Resultados: o interacionismo simbólico busca compreender a ação humana em grupo e acredita que os indivíduos ajam tendo como base os significados que as coisas têm para eles. Esta é uma construção social que se desenvolve na medida em que as pessoas agem e interagem consigo mesmas e com os outros. A continuidade do cuidado, trabalhada a partir das dimensões relacional, informacional e de gestão, coaduna com o proposto, por representar um produto social resultante das interações entre os envolvidos no cuidado, que se expressa segundo sua ação e reação, como uma constante para um conjunto de práticas de diferentes profissionais que buscam garantir o cuidado integral e coerente ao usuário.

Conclusão: este trabalho possibilitou apresentar a continuidade do cuidado, dentro do contexto das interações sociais, não como algo concreto, mas como um objeto social decorrente do agir de cada um dos envolvidos com o cuidado, embora, ainda assim, se perceba a necessidade de sensibilizar profissionais e usuários a respeito de seu significado e intencionalidade no agir da cada um.

DESCRITORES: Continuidade da assistência ao paciente. Assistência integral à saúde. Gestão em saúde. Equipe de assistência ao paciente. Segurança do paciente.

\section{CONTINUITY OF CARE AND THE SYMBOLIC INTERACTIONISM: A POSSIBLE UNDERSTANDING}

\begin{abstract}
Objective: to look for an understanding through the co-substantiation between the concept of continuity of care and the theoretical reference of the symbolic interactionism, in the sense of adding a possibility to strengthen it as an indispensable concept to the care provided to the health system users and to incite a necessary reflection regarding this care.

Method: a theoretical-reflective study, organized in four sections, about the continuity of care analyzed through the symbolic interactionism referential.

Results: the symbolic interactionism intends to understand the human action in a group and believes that individuals act based on the meanings that the things have for them. This is a social construction that develops as people act and interact among themselves and with others. The continuity of care, seen from the relational, informational and management dimensions, is in line with the proposed, since it represents a social product that results from the interactions between those involved in care, which is expressed according to their action and reaction, as a constant for a set of practices of different professionals that intend to guarantee the comprehensive and coherent care to the user.

Conclusion: this study has made it possible to present the continuity of care within the social interactions context, not as something concrete, but as a social object arising from the actions of each of those involved in care, although it is still possible to perceive the need to sensitize professionals and users about their meaning and intentionality in acting.
\end{abstract}

DESCRIPTORES: Continuity of patient care. Comprehensive healthcare. Health management. Patient Care Team. Patient safety. 


\section{INTRODUÇÃO}

Condições de saúde que necessitam acompanhamento de longo prazo são um grande desafio para os prestadores de cuidado em todo o mundo. Tal aspecto se liga ao fato da ocorrência de um maior número de pessoas com multimorbidades, ${ }^{1-2}$ bem como ao incremento das taxas de expectativa de vida. Deste modo, alcançar o maior grau de conectividade entre os cuidados ao longo do tempo representa o ideal percorrido pelos sistemas de saúde $^{1}$, uma vez que os usuários são cada vez mais assistidos por uma variedade de profissionais distribuídos em uma ampla rede assistencial. ${ }^{3}$

Em vista disso, tem ocorrido, nos últimos anos, um crescente interesse pelo conceito de continuidade do cuidado. Contudo, esse é um termo de difícil definição, em grande parte pela falta de um consenso sobre ele, mas, principalmente, porque a continuidade em si é um aspecto do cuidado sentido em especial pelo usuário, ou seja, como ele experiencia a progressão de um cuidado coeso, coordenado e conectado. ${ }^{4}$

Este cuidado, vivido pelo usuário, tem caráter multidimensional, o que significa que depende de um adequado fluxo de informações, boas habilidades interpessoais e boa coordenação dos cuidados, ${ }^{5}$ além do compromisso individual do usuário com a sua própria saúde. Depende da ação-interação entre os envolvidos no cuidado e precisa ser considerado tanto na perspectiva do usuário, quanto dos distintos profissionais de saúde. ${ }^{1,6}$

Acredita-se, portanto, que a ação-interação entre os envolvidos seja central para a continuidade do cuidado, representando o contexto em que esta se desenvolve e se manifesta. Os indivíduos produzem ações de cuidados singulares e, ao interagirem, compartilham e interpretam perspectivas, compreendem as expectativas mutuamente, coordenam suas ações e conduzem o cuidado.

Desta forma, sua compreensão pode ser facilitada pelo referencial teórico do Interacionismo Simbólico, o qual entende que as ações dos indivíduos são desenvolvidas segundo as interações entre as pessoas que, ao interpretarem e definirem as situações, agem no contexto social onde estão inseridas. ${ }^{\text {? }}$

Este referencial possibilita a compreensão da ação social humana, não sua causa estritamente, mas a história da ação, as inúmeras decisões e escolhas que as pessoas fazem quando agem de uma maneira ou de outra. A interação é sempre importante, pois é ela que conduz o caminho do indivíduo. ${ }^{7}$
Compreender a continuidade do cuidado, sob a perspectiva de como a ação humana se desenvolve, auxilia sua interpretação subjetiva como a expressão de um produto social. Considerando o exposto, tem-se por objetivo, neste trabalho, buscar um entendimento pela consubstanciação entre o conceito de continuidade do cuidado ao referencial teórico do interacionismo simbólico, no sentido de agregar uma possibilidade de fortalecê-lo como um conceito indispensável ao cuidado prestado aos usuários do sistema de saúde e provocar uma necessária reflexão sobre o mesmo.

\section{CONSUBSTANCIANDO CONTINUIDADE DO CUIDADO AO INTERACIONISMO SIMBÓLICO}

A continuidade do cuidado, prevista no cenário brasileiro, pela Lei Orgânica da Saúde, procura estabelecer a conexão necessária aos diversos cuidados recebidos pelo usuário ao longo do tempo. Ressalta-se que o período de tempo em questão pode variar de acordo com as demandas de saúde existentes. Necessita da interação e comprometimento dos profissionais de saúde, usuário e família para que os objetivos e metas sejam significativamente compartilhados, garantindo, deste modo, a consistência, a coerência e a conexão entre as ações de cuidado.

Percebe-se, portanto, seu caráter subjetivo, uma vez que não pode ser traduzida como um atributo pessoal ou um objeto em si, mas como um produto social decorrente da ação-interação entre os envolvidos que se manifesta, principalmente, na forma como o indivíduo sente a integração dos cuidados ao longo do tempo. ${ }^{1,4-5,8}$

Para facilitar a compreensão da busca pelo entendimento entre a continuidade do cuidado e o interacionismo simbólico, optou-se por trabalhar o termo "continuidade" pelas dimensões proposta por Reid, McKendry e Haggerty: ${ }^{5}$ continuidade relacional, de gestão e informacional e, em cada uma delas, buscou-se relacionar os conceitos do interacionismo simbólico. Posteriormente, parte-se para uma visão geral da justaposição desses referenciais para mostrar os aspectos particulares do interacionismo que elucidam como a ação humana para a continuidade do cuidado se desenrola internamente no indivíduo. Vale ressaltar que essa é uma divisão única e exclusivamente didática, pois todas as dimensões da continuidade, assim como as interações sociais que propõem o referencial teórico, são indissociáveis e mantêm uma relação de dependência entre elas, o que poderá ser percebido durante a leitura. 
Para tanto, faz-se necessário um breve esclarecimento dos aspectos gerais deste, apresentados aqui em quatro ideias principais. Primeiro, para o interacionismo simbólico, a interação social é central. Os interacionistas entendem o ser humano como uma pessoa social que, ao interagir, constrói a sociedade da qual faz parte, ou seja, os indivíduos são criados pela interação igualmente como a sociedade o é. ${ }^{7}$

A ação humana não deve ser compreendida única e exclusivamente como um resultado apenas da interação com os outros indivíduos, pois, também, resulta da interação individual que cada pessoa faz dentro de si. Com isso, são reveladas a segunda e terceira idéias, nas quais o interacionismo simbólico entende o ser humano como um ser pensante e que define o ambiente onde atua. O ambiente existe, mas é nossa definição sobre ele que é importante. Estas definições não ocorrem ao acaso, resultam de constantes interações sociais e individuais. $\mathrm{O}$ ambiente com o qual o indivíduo age e interage é simbólico. Os símbolos são produzidos em interação e podem ser ou não alterados no curso da interação. ${ }^{7}$ A quarta ideia refere-se à causa da ação humana, a qual é entendida como resultado do que está ocorrendo na situação atual porque se desenrola na presente interação social, no presente pensamento e na presente definição. O passado influencia as ações principalmente porque pensamos sobre ele e o aplicamos para definir a situação atual. ${ }^{7}$

Para melhor compreensão da ação humana, a teoria interacionista se embasa nas seguintes premissas: as pessoas orientam suas ações em direção às "coisas" em razão do que representam para elas. $\mathrm{O}$ significado de tais coisas é consequência da interação social que cada qual mantém com seu próximo, e estes significados são manipulados e modificados na medida em que o processo interativo entre as pessoas se desenvolve. ${ }^{7,9-10}$

Deste modo, acredita-se que profissionais e usuários ajam em relação ao cuidado, ancorados no que este representa para ambos. A ação-interação com o outro permite aos indivíduos significar e ressignificar suas perspectivas em relação ao cuidado e, assim, progressivamente, estas perspectivas vão conduzindo e auxiliando a tomada de decisão acerca do atendimento em saúde.

Ao analisar a continuidade do cuidado, sob a perspectiva do interacionismo simbólico, entendese a continuidade relacional como um conceito que se aproxima mais intimamente da interação entre profissionais e usuários/família. A continuidade de gestão relaciona-se mais fortemente com a interação dos diferentes profissionais entre si e a continuidade informacional, como um símbolo significante para ambas as interações. $\mathrm{O}$ resultado da articulação natural entre todas é o produto social experenciado pelo usuário. Destaca-se que, em determinados contextos, um tipo de continuidade pode estar mais presente do que outro, bem como todos podem ser necessários em uma mesma situação.

\section{Continuidade relacional}

Na perspectiva do usuário, a continuidade do cuidado pode ser definida como uma relação terapêutica contínua de cuidado entre ele e um ou mais profissionais de saúde. ${ }^{11}$ Sob este ponto de vista, é denominada na literatura como continuidade relacional, sendo considerada uma ponte tanto dos cuidados passados com o atual, como também fornece um elo para o cuidado futuro. A continuidade relacional reconhece a importância do conhecimento do usuário como uma pessoa. ${ }^{5,8}$ Este tipo de continuidade permite estabelecer uma relação de confiança, compreensão mútua, sensação constante de responsabilidade para com o paciente e um conhecimento acumulado sobre ele, ${ }^{5,8}$ o que é possível por meio de ações e interações entre profissional e usuário.

Na prática, a continuidade do cuidado se inicia pela interação do usuário com um profissional da saúde, a quem ele procura para um primeiro atendimento que, por sua vez, fará outras interações para dar seguimento do cuidado ao usuário. No entanto, não se pode esquecer da responsabilidade do próprio usuário com sua saúde neste processo.

Ele não pode ser entendido como passivo para a continuidade do cuidado, devendo assumir seu lugar de protagonista, com participação ativa, a fim de efetivar seu acompanhamento em saúde de maneira contínua, conexa e congruente. Deste modo, a continuidade do cuidado precisa ser visualizada como um contrato de atitude que reside em uma relação de "mão dupla" entre o usuário e os profissionais de saúde, que é sustentado pela confiança e responsabilidade dos cuidadores para com o usuário ${ }^{12-13}$ e do usuário para consigo mesmo: é a ação-interação com o outro e consigo.

Não há cuidado continuado se o usuário e/ou a família não tomarem para si a responsabilidade com sua própria saúde. É preciso ainda que os profissionais de saúde, ao se assumirem comprometidos com o cuidado, compartilhem com o usuário e a família o significado da proatividade como uma prática necessária ao cuidado de si e a continui- 
dade do cuidado. Essa é uma ação desejável nos profissionais de saúde para solidificar e sustentar a continuidade como uma reação dos usuários para com seu próprio cuidado.

Tal atitude condiz com o proposto pelo interacionismo simbólico de que as pessoas se relacionam de tal forma que o ato de um indivíduo gera um estímulo no outro, que reage e se adapta. As reações e adaptações do segundo indivíduo, por sua vez, convergem em estímulos mutuamente no primeiro indivíduo, fazendo com que este se modifique e inicie um ato distinto. ${ }^{10}$

Embora o contato contínuo do usuário com um mesmo profissional de saúde apresente inúmeras vantagens, é preciso considerar a continuidade em um caráter multiprofissional, uma vez que a complexidade do processo saúde-doença exige, em certos momentos, saberes e cuidados distintos. Portanto, a ação-interação é importante tanto entre profissionais, usuários e familiares, como também entre estes profissionais em si, o que é possível com a continuidade de gestão.

\section{Continuidade de gestão}

$\mathrm{Na}$ perspectiva dos profissionais, a continuidade do cuidado é a expressão da entrega de um serviço contínuo, que ocorre pela integração, coordenação, partilha de informações e comunicação adequada entre os diferentes cuidadores. ${ }^{11,14}$ Neste caso, ela é identificada como continuidade de gestão, que garante que os cuidados recebidos por diferentes profissionais sejam conectados de forma coerente. Este tipo de continuidade é geralmente focado em um plano de gestão para um problema de saúde específico. ${ }^{8}$

A continuidade de gestão envolve, além da interação entre diferentes profissionais em uma equipe interdisciplinar, também a interação entre diferentes serviços e até mesmo instituições. Ela garante que os cuidados em saúde se completem, sendo necessário, para tanto, que os distintos profissionais e serviços trabalhem de forma integrada e articulada, o que é possível por meio de protocolos e planos de gestão do cuidado coerentes com as necessidades do usuário. ${ }^{5,8}$

Nesse sentido, a continuidade do cuidado é essencial para um atendimento qualificado. Sem ela, é pouco provável que o cuidado seja clinicamente eficaz, seguro, personalizado, eficiente, econômico e integral. Falhas nesse sentido, ou descontinuidade do cuidado, podem pôr a saúde do indivíduo em risco, causar duplicidade de con- dutas e adicionar custos evitáveis para o sistema de saúde e assistência social. ${ }^{6,8,13}$

Neste aspecto, pode-se pensar a continuidade do cuidado como um símbolo significante para o atendimento das expectativas de saúde de um indivíduo e do sistema de saúde como um todo. No interacionismo, o símbolo é considerado um conceito central, sem o qual a interação social se torna impraticável. Um símbolo é qualquer objeto social utilizado para representar alguma coisa. Pode ser tanto um objeto físico, quanto uma atitude pessoal ou palavras, mas ele só pode ser considerado simbólico ou significante quando houver uma representação ou intencionalidade, ou seja, quando um gesto que contém uma ideia por trás de si provoca essa mesma ideia no outro, fazendo com que este segundo indivíduo se identifique com ele. .,9-10,15 $^{-15}$

Para uma ação de cuidado ser continuada de maneira que seja complementar e coerente ao cuidado anterior ou futuro, os símbolos utilizados durante as ações-interações necessitam ter significados compartilhados para ambos os indivíduos, ou seja, a ação e a intenção do cuidado do primeiro indivíduo necessitam expressar o mesmo sentido para o segundo indivíduo. E, desta maneira, efetiva-se a conexão entre ambas as ações para a continuidade do cuidado ao usuário.

Os significados atribuídos a determinados símbolos podem ser expressos de formas diferentes em contextos diversos. Para a continuidade do cuidado, as palavras ganham lugar de destaque. É por meio delas que o conhecimento da informação a respeito do histórico de saúde do usuário é possível. Deste modo, a informação também é considerada como um elemento central.

Por meio da informação, os distintos profissionais podem articular-se entre si para elaborar um plano de gestão do cuidado coerente com o usuário, bem como este usuário poderá continuar seu próprio cuidado de posse das informações compartilhadas. Tais aspectos são viáveis pela continuidade informacional.

\section{Continuidade informacional}

A continuidade informacional possibilita o conhecimento de eventos e circunstâncias prévias do usuário, sejam elas a respeito de condutas, recomendações, situações da vida diária, resultados laboratoriais ou até mesmo de um cuidado informal. ${ }^{5}$ Percebe-se, então, a necessidade do uso da informação na forma de bons registros para a transferência do conteúdo desta, já que esta é a lin- 
guagem comum que liga o cuidado anterior ao atual e futuro. Neste aspecto, a carência de um sistema de informação interligado entre todos os níveis de atendimento à saúde é considerada fator limitante à continuidade do cuidado.

Para a adesão ao tratamento e continuidade pelo próprio usuário e família, além da sua corresponsabilidade, as informações e orientações compartilhadas também necessitam de um sistema de registro e consulta acessível para o sucesso e seguimento da terapêutica proposta. ${ }^{16}$

É preciso esclarecer que a simples transferência de informações, por si só, não garante a Continuidade do Cuidado. Para tal, estas informações necessitam ser interpretadas ${ }^{8}$ e ter seus significados compartilhados para definir a situação e guiar a tomada de decisão. Portanto, ressalta-se o uso simbólico das palavras como meio de viabilizar a comunicação/linguagem, ou seja, na continuidade do cuidado, conforme proposto pelo interacionismo, as palavras são símbolos que, por terem significados atribuídos pelos indivíduos, possibilitam a comunicação, a compreensão e a transferência de informações.

O próprio usuário e o profissional são considerados fontes de informações, uma vez que utilizam a memória como um arquivo itinerante. Este aspecto pode parecer pouco científico, porém, é por meio dele que as impressões pessoais são transferidas, já que estas informações são pouco valorizadas nos registros formais e conhecidas apenas quando os envolvidos interagem e se comunicam. O reconhecimento dos valores, preferências, contexto social e de apoio de um usuário também tem impacto sobre a adequação e adesão do plano de cuidado. ${ }^{5}$

Deste modo, a ação-interação com o outro, mais uma vez, ganha lugar de destaque, uma vez que, nesta conjuntura, o acesso a estas informações só é possível por meio dela, tanto no sentido da continuidade de gestão em que estas informações precisam ser compartilhadas para guiar o plano de cuidados, quanto na continuidade relacional por meio da qual são identificadas e, posteriormente, reiteradas, para a condução e adesão ao cuidado.

É preciso considerar que cada indivíduo carrega consigo convicções e perspectivas próprias em relação às suas ações de cuidado e, durante o curso de uma ação-interação, estas ações podem ser alteradas ou mantidas. Isso acontece porque a ação humana é resultante não apenas da interação com os outros, mas depende de um processo reflexivo interno de cada indivíduo. Deste modo, a comunicação fornece meios para os indivíduos debaterem os significados, favorece a compreensão mútua e, por conseguinte, guia suas condutas.

De acordo com o interacionismo simbólico, os seres humanos dão sentido aos símbolos e expressam as coisas por meio da linguagem/comunicação. ${ }^{15}$ Entende-se que o significado seja uma condição que surge como resultado da ação-interação entre as pessoas, não uma característica intrínseca do objeto. ${ }^{10,15}$ Assim, o reconhecimento do que é ou não significante para o usuário e para o profissional é possível quando eles interagem, compartilham informações, perspectivas e expectativas acerca do cuidado.

\section{Uma visão geral da justaposição dos conceitos}

Antes de iniciar, é importante esclarecer que nem toda interação é simbólica. A interação não simbólica ocorre quando o ser humano responde diretamente aos gestos ou ações do outro - uma resposta involuntária. A interação só é simbólica quando o indivíduo interpreta os gestos do outro e age com base no significado desta interpretação. ${ }^{9}$

Assim, quando há interação sem compartilhamento de significados, as ações dos profissionais de saúde e usuários em relação ao cuidado podem caminhar para lados opostos, gerando lacunas ou descontinuidade do cuidado. Tal aspecto pode apresentar consequências ao usuário pela possibilidade de perda da coerência no plano de cuidado.

Percebe-se que, segundo esta abordagem teórica, a ação humana não é uma mera resposta aos estímulos do ambiente, uma resposta sem reflexão. Ao contrário, o indivíduo observa as coisas à sua volta, atribuindo significados às ações dos outros para delinear sua conduta à luz desta interpretação. ${ }^{9}$ Isso porque a ação social de um indivíduo não é um produto único da interação com o outro, mas também da interação que cada um tem consigo mesmo, da autorreflexão.

O processo reflexivo e interpretativo que cada indivíduo tem consigo mesmo é possível por meio da ação da mente, quando ele utiliza o self. A mente é produto da comunicação que ocorre por meio de uma conversação de gestos de um processo social ou contexto de experiência, ou seja, a mente é uma relação do organismo com a situação que se realiza por meio de uma série de símbolos. ${ }^{7,-10,15}$

O self representa o processo social interno de cada um. Isso significa dizer que o indivíduo é um objeto de sua própria ação. Ele pode perceber-se, ter concepções de si mesmo, comunicar-se consigo mesmo e agir em relação a si mesmo. ${ }^{7,9-10,15}$ Deste modo, ao tomar uma decisão em relação ao cuidado, 
a ação de um profissional pode ter um significado diferente da ação do mesmo cuidado para outro profissional, principalmente, quando se refere a categorias profissionais diferentes. Ambas as ações, quando negociadas e compreendidas internamente por estes profissionais, ao interagirem, se completam e se convergem em cuidado integral ao usuário, pois um continua a ação do outro.

O mesmo ocorre no que concerne à interação com o usuário que, ao interagir consigo mesmo, continua a ação para o seu cuidado, isto por compartilhar o significado daquela informação e prática como importante para alcançar os resultados esperados para sua saúde. Tal aspecto depende, é claro, do contexto em que ele pode assumir ou não um papel ativo neste processo como, por exemplo, na continuidade do cuidado, durante um planejamento de alta, quando esse usuário precisará continuar o cuidado em casa sem necessidade de envolvimento direto de outros profissionais.

É por meio da ação da mente, dentro de um contexto de ações-interações, que cada profissional e usuário definem para si como sua ação singular de cuidado deve ser conduzida. É, portanto, um processo constante de fazer indicações para si mesmo a respeito dos objetos em seu ambiente e, especialmente, sua utilização ajuda o indivíduo a alcançar seus objetivos. ${ }^{7}$ A condução do cuidado entre os profissionais e usuários, para ser caracterizado como contínuo, depende proporcionalmente da negociação interna que cada um faz dentro de si. É uma autorreflexão que considera tanto a perspectiva do outro, quanto a própria perspectiva. ${ }^{7}$

Esta autointeração auxilia profissionais a ter condutas social e cientificamente esperadas, como se fosse um mecanismo de controle moral do indivíduo; auxilia os usuários a dar continuidade ao cuidado para consigo mesmo, embasados nos significados das condutas dos profissionais.

Por exemplo, numa situação em que um usuário com suspeita de um quadro infeccioso transmissível dá entrada em uma unidade de internação que, em um primeiro momento, só dispõe de vagas em quartos compartilhados, o profissional de saúde responsável naquele momento, com posse das informações sobre o quadro do paciente, ao interagir com os envolvidos e consigo mesmo, age no sentido de remanejar os demais pacientes e providenciar o isolamento necessário até que se confirme ou não a hipótese diagnóstica.

Esta atitude promove a continuidade do cuidado tanto para ele, quanto para os demais usuários, porque o profissional em questão, diante da possi- bilidade de ser ou não um quadro infeccioso, utiliza o self e reflete que a possibilidade de ignorar como verdadeira a hipótese diagnóstica poderia pôr em risco a segurança dos demais pacientes da unidade e, por outro lado também, não deixa o novo paciente sem atendimento e acompanhamento.

Da mesma forma, o profissional ou usuário utiliza o self em um contexto da experiência para significar ou ressignificar uma ação e, com isto, conduzir a um caminho ou a outro o seu agir. A capacidade de se antecipar a um evento, com a intenção de promover o cuidado coerente, compreender as ações do outro e compartilhar a sua conduta segundo sua própria mente, é alcançada quando o indivíduo se põe no lugar do outro.

De acordo com o interacionismo simbólico, colocar-se no lugar do outro é essencial à comunicação simbólica e ao desenvolvimento de si, sendo considerada uma das mais importantes atividades mentais. Esta característica permite ao indivíduo ensinar, aprender, cooperar, agir moralmente, ter simpatia, influenciar, ajudar, proteger-se, controlar suas próprias ações e perceber as consequências de suas ações. É uma das partes mais importantes do que se classifica como inteligência social. ${ }^{7}$

Representa um mecanismo básico por meio do qual as interações acontecem. É a capacidade não apenas de se colocar no lugar do outro, ou imaginar o mundo pela perspectiva do outro; mas de antecipar como esse outro irá pensar, sentir ou reagir. Permite compreender como e por qual motivo os indivíduos agem de uma maneira ou de outra. ${ }^{7,17}$ Assim, é possível conceber a continuidade do cuidado como uma ação humana decorrente das ações-interações e autointeração dos envolvidos.

Nessa perspectiva, destaca-se que os seres humanos se envolvem em um contínuo fluxo de ação que pode ser tanto aberta, quanto encoberta, influenciada por decisões tomadas, resultantes da interação social e com o self. Cada ação tem uma história, que é direcional. As decisões e definições que os indivíduos tomam é que mudam ou não a direção de suas vidas. ${ }^{7}$

Do mesmo modo, a continuidade do cuidado representa um fluxo de ação uniforme e progressivo de cuidados ao longo do tempo e do espaço. Isto envolve a ação-interação humana em um determinado espaço físico ou situação, tanto em momento atual, quanto de um período de tempo para outro, de um ambiente para outro e/ ou de uma disciplina para outra. ${ }^{5,18}$ A ação é direcionada de acordo com as metas e objetivos que os indivíduos determinam como importantes para eles. 
Assim, a ação humana individual, no processo de interação simbólica, conduz a uma ação conjunta, que se refere ao comportamento cooperativo, desenvolvido pela percepção de cada indivíduo sobre a intenção do outro, construindo, desta forma, uma resposta baseada naquela intenção. ${ }^{9,15}$ Consiste na interligação das respectivas ações individuais, no entanto, a ação conjunta é distinta, não podendo ser considerada uma mera somatização de ações isoladas dos indivíduos, pois cada pessoa ocupa uma posição diferente e age segundo esta posição, envolvendo-se em um ato separado e distinto., ${ }^{9,15}$

Do mesmo modo, percebe-se a continuidade do cuidado como fruto de uma ação conjunta, que se caracteriza não como mera soma de múltiplos cuidados parciais, mas como uma articulação, reflexiva, negociada, compartilhada, significativa e coerente das diversas ações singulares de cuidados por parte dos envolvidos no contexto das interações em que ocorre e se manifesta na forma como o usuário percebe esta ação conjunta do cuidado.

\section{CONCLUSÃO}

Refletir acerca da continuidade do cuidado é imprescindível, dada a realidade posta das transformações sociais, econômicas, demográficas e epidemiológicas do mundo atual. Deste modo, torna-se uma necessidade pensar na capacidade que um sistema de saúde tem para dar conta das demandas de cuidados dos usuários.

No cenário do sistema de saúde brasileiro, apesar da continuidade do cuidado estar prevista na Lei Orgânica da Saúde, ainda não é uma realidade totalmente disponível aos usuários. A conformação das redes de atenção surge com o objetivo de combater as fragmentações e fragilidades e, apesar dos seus avanços, ainda é preciso ir além para que a continuidade do cuidado se concretize como uma ação sistematizada na práxis.

Este artigo procura compreendê-la dentro do contexto das interações sociais, não como algo concreto, palpável, mas como um produto social decorrente do agir de cada um dos envolvidos com o cuidado. Destaca-se que este processo envolve uma série de aspectos que necessitam ser levados em consideração para que a continuidade se apresente como um cuidado coerente ao usuário. Ou seja, ela é resultado de um conjunto de práticas que dependem da comunicação eficaz, bom relacionamento entre profissionais e usuários, trabalho interdisciplinar, articulação entre prestadores, compartilhamento de informações e significados, adequada coordena- ção e integração do cuidado entre todos os níveis de atendimento em saúde, bem como depende da concepção do usuário neste processo não apenas como passivo à continuidade, mas também como ativo e responsável por sua saúde.

Contudo, a continuidade do cuidado ainda é um conceito pouco disseminado na prática assistencial e, em decorrência de sua subjetividade, compreendê-la como uma ação social auxilia a reflexão acerca do tema e a fortalece como um conceito essencial do cuidado ao usuário. Ressalta-se a necessária sensibilização dos profissionais e usuários a respeito de seu significado e intencionalidade no agir da cada um. Para tanto, este é um tema que suscita mais discussões e pesquisas, principalmente, no contexto brasileiro, que se apresenta discreto e incipiente em comparação aos países mais desenvolvidos.

\section{REFERÊNCIAS}

1. Hill MK, Twiddy M, Hewison J, House AO. Measuring patient-perceived continuity of care for patients with long-term conditions in primary care. BMC Family Practice [Internet]. 2014 [cited 2016 Aug 23]; 15(191):1-8. Available from: http:// bmcfampract. biomedcentral.com/articles/10.1186/s12875-0140191-8

2. Barnett K, Mercer SW, Norbury M, Watt G, Wyke S, Guthrie B. Epidemiology of multimorbidity and implications for health care, research, and medical education: a cross-sectional study. Lancet [Internet]. 2012 [cited 2016 Aug 20]; 380(9836):37-43. Available from: http://www.thelancet.com/journals/lancet/ article/PIIS0140-6736(12)60240-2/abstract

3. Tarquini R, Coletta D, Mazzoccoli G, Gensini GF. Continuity of care: an italian clinical experience. Intern Emerg Med [Internet]. 2013 [cited 2016 Aug 20]; 8(7):595-9. Available from: http:/ /link.springer. com/article/10.1007/s11739-012-0808-7

4. Freeman GK, Woloshynowych M, Baker R, Boulton M, Guthrie B, Car J, et al. Continuity of care 2006: what have we learned since 2000 and what are policy imperatives now? report for the national co-ordinating centre for NHS services delivery and organisation R \& D (NCCSDO) [Internet]. 2007 [cited 2016 Aug 20]. Available from: http://www.netscc.ac.uk/hsdr/ files/project/SDO_FR_08-1609-138_V01.pdf

5. Reid RJ, McKendry R, Haggerty J. Defusing the confusion: concepts and measures of continuity of health care [Internet]. 2002 [cited 2016 Aug 20]. Available from: http://www.cfhifcass.ca/Migrated/ PDF/ResearchReports/CommissionedResearch/ cr_contcare_e.pdf

6. Freeman G, Hughes J. Continuity of care and the patient experience. [Internet]. 2010 [cited 2016 Aug 10]. Available from: http://www.kingsfund.org.uk/sites/files/ 
kf/field/field_document/continuity-care-patientexperience-gp-inquiry-research-paper-mar11.pdf

7. Charon JM. Symbolic interacionism: an introduction, an interpretation, an integration. New Jersey (US): Prentice-Hall; 2010.

8. Haggerty JL, Reid RJ, Freeman GK, Starfield BH, Adair CE, Mckendry R. Continuity of care: a multidisciplinary review. BMJ [Internet]. 2003 [cited 2016 Jul 13]; 327(7425):1219-1221. Available from: http:/ / www.bmj.com/content/327/7425/1219.full

9. Blumer H. Symbolic interacionism: perspective e method. Berkeley (US): University of Califórnia; 1969.

10. Carvalho VD, Borges LO, Rêgo, DP. Interacionismo simbólico: origens, pressupostos e contribuições aos estudos em psicologia social. Psicol Cienc Prof [Internet]. 2010 [cited 2016 Jul 13]; 30(1):146-61. Available from: http://www.scielo.br/pdf/pcp/ v30n1/v30n1a11.pdf

11. Gulliford M, Naithani S, Morgan M. What is "continuity of care"? J. Health Serv Res Policy [Internet]. 2006 [cited 2016 Jul 13]; 11(4):248-50. Available from: http://www.ncbi.nlm.nih.gov/ pubmed/17018200

12. Banahan BFJ, Banahan BFI. Continuity as an attitudinal contract. J Fam Pract [Internet]. 1981 [cited 2016 May 05]; 12(4):767-8. Available from: https:/ / www.researchgate.net/publication/15994277_ Continuity_as_an_attitudinal_contract

13. Cornwell J, Levenson R, Sonola L, Poteliakhoff E. Continuity of care for older hospital patients: a call for action. The king's Fund [Internet]. 2012 [cited 2016 May 25]. Available from: http://www.kingsfund. org.uk/sites/files/kf/field/field_publication_file/ continuity-of-care-for-older-hospital-patientsmar-2012.pdf

14. Tandjung R, Rosemann T, Badertscher N. Gaps in continuity of care at the interface between primary care and specialized care: general practitioners' experiences and expectations. Int J Gen Med [Internet]. 2011 [cited 2016 Apr 25]; 4:773-8. Available from: https:/ / www.dovepress.com/gaps-in-continuityof-care-at-the-interface-between-primary-care-ands-peer-reviewed-article-IJGM

15. Lopes CHAF, Jorge MSB. Interacionismo simbólico e a possibilidade para cuidar interativo em enfermagem. Rev Esc Enferm USP [Internet]. 2005 [cited 2016 Apr 25];39(1):103-8. Available from: http:/ / www.ee.usp. br/reeusp/upload/pdf/52.pdf

16. Morales CLP, Alexandre JG, Prim S, Amante LN. A comunicação no período perioperatório sob a ótica dos pacientes submetidos à cirurgia bariátrica. Texto Contexto Enferm [Internet]. 2014 [cited 2016 Oct 20]; 23(2):347-55. Available from: http:/ / www.scielo.br/ pdf/tce/v23n2/pt_0104-0707-tce-23-02-00347.pdf

17. Carvalho LS, Silva, CA, Oliveira, ACP, Camargo, CL. $\mathrm{O}$ interacionismo simbólico como fundamentação para pesquisas de enfermagem pediátrica. Rev Enferm UERJ [Internet]. 2007 [cited 2016 Apr 25]; 15(1):119-24. Available from: http://www.facenf.uerj.br/v15n1/ v15n1a19.pdf

18. Uijen AA, Schers HJ, Schellevis FG, Bosch WJHMVD. How unique is continuity of care? a review of continuity and related concepts. Fam Pract [Internet]. 2012 [cited 2016 Aug 25]; 29(3):264-71. Available from: https://academic.oup.com/fampra/article-lookup/ doi/10.1093/fampra/cmr104 